\title{
Multiscale Approaches for the Simulation of Optoelectronic Devices
}

\author{
Matthias Auf der Maur \\ University of Rome Tor Vergata, Rome, Italy \\ E-mail:auf.der.maur@ing.uniroma2.it \\ Received 5 November 2015; Accepted 3 June 2016; \\ Publication 1 August 2016

\begin{abstract}
Multiscale approaches for electronic device simulation have become a subject of high interest during the last decade. In this article we will give an overview on the current activities in the field. We will provide some practical examples from optoelectronics where multiscale simulations can be useful. Basic coupling schemes are discussed, and approaches for the combination of continuous models and models with atomistic resolution are described.
\end{abstract}

Keywords: multiscale simulation, optoelectronic devices.

\section{Introduction}

Historically, multiscale modeling started independently at different times in different fields. In 1976 a simulation of a complex chemical system has been described, coupling wave function based quantum mechanics with a classical molecular mechanics model [18]. In solid mechanics, multiscale methods have become popular since the late 1990 in the field of material science and mechanics and are now well established [35, 30, 53]. Later, and mainly driven by the downscaling trend in microelectronics, multiscale methods started gaining attention also for electronic transport, and subsequently for thermal transport. The first example of coupling a microscopic transport model, in that case based on non-equilibrium Green's functions, with a semiclassical

Journal of Green Engineering, Vol. 5, 133-156.

doi: 10.13052/jge 1904-4720.5349

(c) 2016 River Publishers. All rights reserved. 
drift-diffusion model has been presented in 2003 [39]. Since then, more and more research has been performed towards multiscale device simulations, leading to an increased number of publications in recent years as shown in Figure 1. The figure shows the number of articles where the keyword "multiscale" is appearing in the title together with "simulation" or "model" in title or abstract. Part of these publications specifically treat problems involving electronic or optoelectronic systems, a subset of which can be assumed to actually treat device simulation. Also in thermal modeling multiscale approaches have been developed in recent years [34, 58], although to lesser extent, and similar for optical simulations [41].

Since the term "multiscale" is used in different scientific fields, it assumed slightly different meanings across disciplines. In the mathematical community, for example, it usually indicates problems and associated solution methods, where the solution contains components on multiple time or length scales. Some examples of methods in this field are homogenization [17], heterogeneous multiscale methods [66] or variational multiscale methods [36], where a modified "macroscale" problem containing the effect of the "microscale"

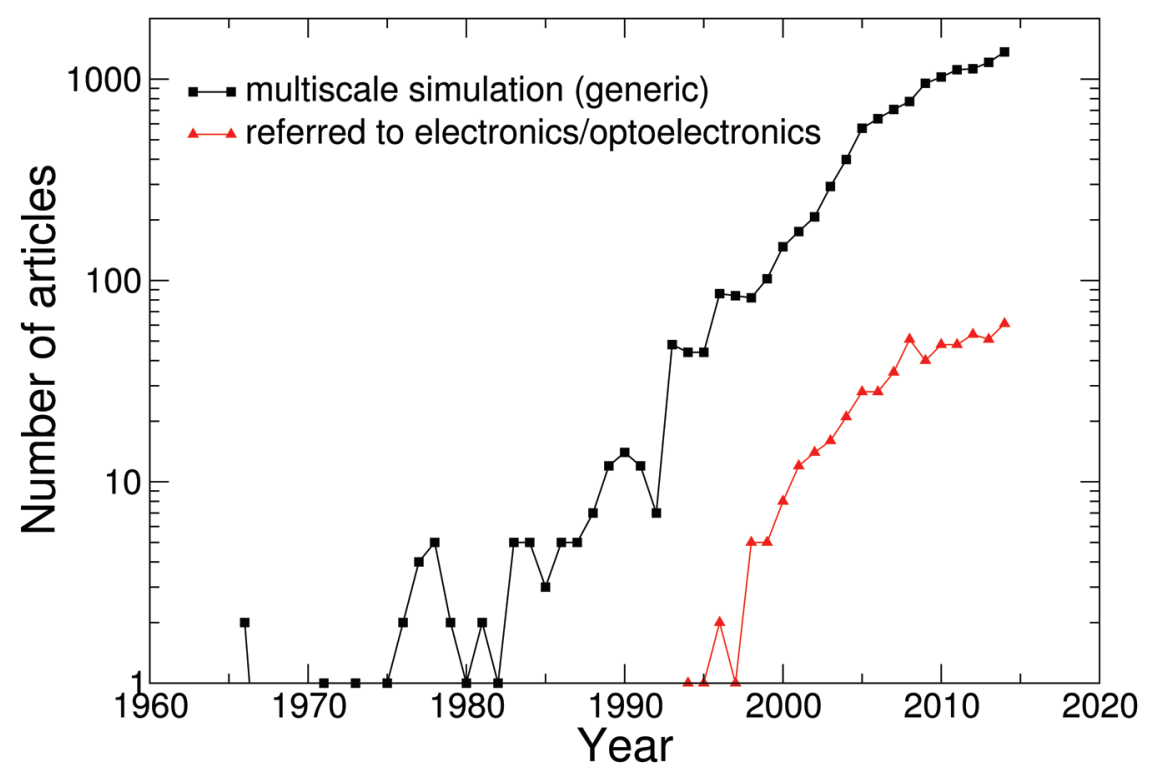

Figure 1 Number of publications on multiscale simulation indexed in Scopus. The black rectangles are for generic multiscale simulation topics (search terms "multiscale" in title and "simulation" or "model" in title/keywords/abstract), the red triangles indicate the same data restricted to items where the terms "electronic", "optoelectronic" or "device" appear. 
features is constructed and solved. In electronic device simulation, which is the object of interest in this article, the term "multiscale" refers to a modeling approach where information regarding microscopic features are embedded into the device scale model. The way this is done strongly depends on the problem to be solved, but often it is understood in such a way that models on different scales are solved concurrently and coupled together explicitly.

The interest in multiscale modeling of electronic devices has been driven on the one hand by the downscaling of the dimensions of classical devices, most of all MOSFETs, and on the other hand by the introduction of nanometer scale, low dimensional functional elements in the active regions. In both cases, the standard simulation approaches based on the solution of the Poisson and the semi-classical drift-diffusion equations looses validity and eventually breaks down. This is due to an increasing importance of quantum mechanical effects, like confinement and coherence, and of increasing deviation from a local equilibrium situation. A review on some of the transport model classes and their relation across scales is given in [9].

To some extent, quantum mechanical effects related to electronic states like confinement can be accounted for by a quantum correction of the semi-classical model. For example, quantum corrected Poisson/drift-diffusion models are readily used in order to correctly include the quantum mechanical carrier densities [26]. Such "Schrödinger/drift-diffusion" approaches provide a more accurate description of the electronic states and thus of the local density of states (DOS), but they still neglect both quantum effects in transport and non-equilibrium effects. While the latter can be resolved by resorting to quantum-corrected Monte Carlo simulations [69], for the former it is necessary to move towards fully quantum mechanical transport theories like Wigner Monte Carlo [57], density matrix based [64] or non-equilibrium Green's functions (NEGF) [24]. In particular NEGF currently enjoys great popularity. However, such models are computationally expensive so that the simulation domain needs to be restricted to small device regions containing possible just the very active region. This is unsatisfactory from an engineering point of view, where the global behaviour of the complete device including e.g. access regions is of interest, and from the fact that a nanometer scale active region is usually embedded in a larger environment which can interact with the properties in the active region in various ways, e.g. by means of mechanical strain or thermal heating. Moreover, it may be desirable or necessary to base the detailed quantum mechanical model for the active region on an atomistic description of the device. While there are several reasons to do so, this certainly further limits the spatial simulation domain. It becomes therefore necessary to 
couple the detailed quantum mechanical or atomistic description, sometimes called micro- or subscale model, to the more approximate models at the higher scale, sometimes denoted as macroscale.

Such a coupling, which may involve models for several different physical entities in a multiphysics setup, should eventually allow to obtain an accurate overall simulation model of a device, to be used for predictive simulations and device optimisation.

In the following we will present an overview on current coordinated actions on multiscale modeling and simulation, introduce the coupling schemes relevant for electronic devices, and show some examples where multiscale approaches can provide valuable insight into device behaviour.

\section{Initiatives in Multiscale Modeling and Simulation}

Since several years, science funding agencies in the US and in Europe regularly open calls specifically referring to multiscale aspects. At the beginning of 2016, the US National Science Foundation (NSF, [1]) has several calls open where multiscale modeling and simulation is playing an inportant role. These are in the fields of Biomechanics and Mechanobiology, design of materials for future applications, and related to high performance computing systems (HPC).

Also the US Army is active in the research in multiscale topics, with special focus on electronic applications. The Army Research Laboratory initiated in 2012 a collaborative research alliance about Multi-Scale Multidisciplinary Modeling of Electronic Materials with the objective "to develop quantitative understanding of materials from the smallest to the largest relevant scales to advance the state of the art in electronic, optoelectronic and electrochemical materials and devices." Areas of interest are indicated as Electrochemical Energy Devices, Hybrid Photonic Devices, and Heterogeneous Metamorphic Electronics, and the program defines a timeframe of 10 years, with the final goal described as "Materials Optimization and Materials by Design".

The European Union has funded already in the 7th Framework Programme (FP7) a number of projects on multiscale modeling. Additionally, projects financed after 2012 in FP7, and new projects on multiscale topics in the framework in H2020, have been gathered in a "Multiscale Materials Modelling Cluster" with the idea of "bringing together European Commission funded projects that have the ambition to develop an open, integrated and multi-purpose numerical nano-design environment" [2]. The cluster includes currently the projects 
DEEPEN (From atom-to-Device Explicit simulation Environment for Photonics and Electronics Nanostructures, http://www.nmp-deepen.eu)

MMP (Multiscale Modelling Platform: Smart design of nano-enabled products in green technologies, http://www.mmp-project.eu),

MoDeNa (MOdelling of morphology DEvelopment of micro- and NAnostructures, http://www.modenaproject.eu),

NanoSim (A Multi-scale Simulation-Based Design Platform for Cost-Effective $\mathrm{CO}_{2}$ Capture Processes using Nano-Structured Materials, http://www.sintef.no/Projectweb/NanoSim),

SimPhoNy (Simulation framework for multi-scale phenomena in micro- and nanosystems, http://www.simphony-project.eu),

ICMEg (The Integrated Computational Materials Engineering expert group, a Coordination and Support Action, http://www.icmeg.eu),

MOSTOPHOS (Modeling Stability of Organic Phosporescent Light Emitting Diodes, http://www.mostophos-project.eu).

The cluster is coming out of a broader initiative, the European Materials Modeling Council (EMMC, http://www.emmc.info), founded in 2012. The council is a community driven bottom-up action in order to connect all existing material modelling activities in Europe, and it has been started due to the observations that on the one hand the European Industries' future is associated with strong modeling capabilities, and on the other hand that the research activities in materials modeling in Europe, which covers variuos fields and includes the multiscale simulation community, needs to be defragmented and brought together. The EMMC therefore has spent a lot of effort during the last years in order to define a vocabulary and classification adapt to modeling and simulation, which is generic and independent of the specific field of application. The outcome of this should help interaction between modeling communities on the one hand, and lead to unified and thus generic simulation 
frameworks on the other. The latest document, including the recommended vocabulary can be found in [25].

A further action regarding multiscale modeling, focused on photovoltaics, has been started in 2015 in the framework of the European COST association. The goal of the COST Action MultiscaleSolar (www.multiscalesolar.eu) is to defragment knowledge and research activities across the Action's member countries and to apply multiscale modeling concepts to next generation photovoltaic concepts, validated at each scale by experiment.

\section{Multiscale Coupling Schemes}

Let us assume a multiscale problem which allows the identification of two different scales. The problem can then be associated with one of two types of setups, depending on how the scales are separated and how the respective models interact. For the discussion here we identify the two scales by the names macro and microscale, which is rather usual in mathematical literature. Note that these terms are abstract and do not refer to specific physical length (or time) scales. The two setups, which can be called overlap and bridge scheme [11, 15], respectively, are shown schematically in Figure 2. The difference between the two setups is that in the overlap scheme the domains where the microscale and macroscale models are applied overlap, while in the bridge scheme the two domains are non-overlapping. Depending on the

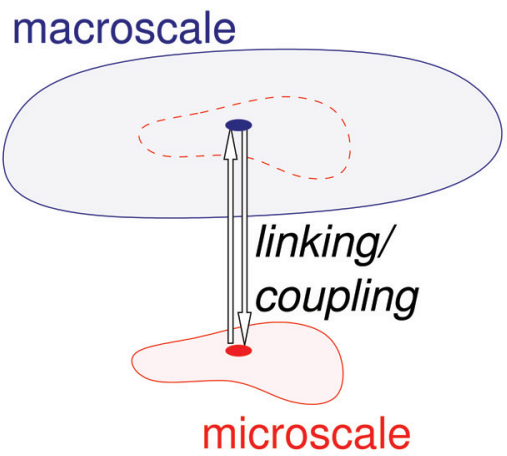

(a)

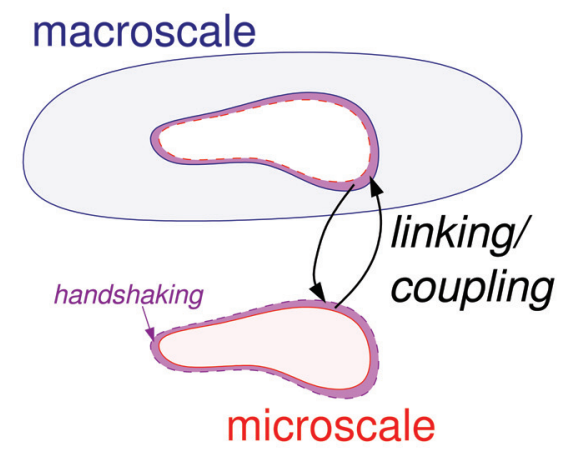

(b)

Figure 2 Overlap (a) and bridge (b) scheme for coupling between models on different scales. Depending on the problem and implementation the bridge scheme can include an overlapping handshaking region. 
implementation and type of problem, the latter may however contain a small overlapping handshaking region at the interface between the two domains.

Typically, in overlap type of problems the macro- and microscale models provide parameters to each other. For example, in a transport simulation the microscale model may provide local mobilities and recombination rates, which are used in a macroscopic model describing particle drift and diffusion. In this sense, the two scales describe different aspects of the overall physical problem. In a bridge scheme, on contrary, the two models typically describe the same physical quantity. Using the same example of particle transport, the macroscale domain might be the part of a device where a model like driftdiffusion provides a good description, while in the microscale domain a more detailed microscopic model is needed. Both models provide as a result the particle current which needs to be fitted together at the inter-domain boundary due to continuity requirements. In such a case, the two scales provide boundary conditions to each other.

A further classification of multiscale setups can be done with respect to the nature of data exchange between the scales. This can happen in one direction only, which is the case for example when the microscale model is providing model parameters to the macroscale model. Such a one way coupling is sometimes called parametric or hierarchic multiscale, while the EMMC vocabulary recommends to use the term linking in this case. The second possibility is a two way coupling, where data exchange happens in both directions. An example of this has been given above, where two transport models are coupled self-consistently, one of them providing driving potentials to the other, which in turn provides an interface current to the former. This is called in literature a fully coupled or concurrent multiscale simulation, while the EMMC vocabulary in this case reccommends simply the term coupling.

The implementation of a multiscale scheme for some physical problem implies the identification and separation of the different scales involved. The way this is done depends largely on the type of problem and models involved. Typical multiscale problems studied in the mathematical literature for example are based on partial differential equations, where different scales are present due to the intrinsic structure of the solution, or due to microscale fluctuations in the parameters $[29,36]$. In this case, there is one mathematical model describing the problem, and the micro- and macroscales correspond to the scale which can or cannot resolve the finestructure of the solution, respectively. The scale separation can be performed for example by a sum decomposition of the solution into two components of which one resolves the microscale features, 
and the solution can be sought in a variational setup with strong connection to finite element methods and numerical stabilization approaches [36].

More generally, the models describing the different scales might be of different nature, leading to heterogeneous multiscale methods [66]. This is the typical type of problem encountered in electronic device modeling. As an example, a particle transport simulation might require the explicit calculation of its parameters from the local microscopic material properties. Such types of setups have been succesfully applied in particular in the field of solid mechanics, e.g. for the calculation of crack propagation and elastic waves $[8,67,70]$, and also in electrostatic analysis [68].

Particular interest is devoted to multiscale setups where the microscale model relies on an atomistic description of the material, while the macroscale model uses a continuous media description. Such approaches have been followed in solid mechanics problems as shown in the references given before, but during the last decade they became of relevance also for electronic devices $[12,15,33,48]$.

\section{Multiscale Problems in Optoelectronics}

In this section we present a few examples of modeling problems in optoelectronics, where multiscale simulation approaches have already been applied or which lend themselves for a multiscale description.

\subsection{Optical Properties in III-nitride LEDs}

III-nitride based light emitting diodes (LEDs) consisting of InGaN quantum wells (QW) embedded in GaN are currently the most efficient solid state light sources for lighting applications [62]. Although these devices are commercially available, there are still some fundamental issues related to their quantum efficiency of light generation to be solved. In particular, the efficiency drops when moving from blue emitters with low indium content towards green ones with high indium content, and it also drops with increasing injecting current. The former is known as "green gap" [61], the latter as "droop" [63]. Efficiency droop is believed to be mostly related to Auger recombination, and a detailed simulation of droop therefore requires a microscopic model for Auger recombination beyond the semi-classical formulas usually used in simulations. The green gap, on the other hand, was recently found to be associated with the random statistical fluctuations of the InGaN alloy, which is most naturally described by means of models based on an atomistic 
description [14]. In fact, InGaN/GaN based LEDs have been extensively studied by combining atomistic and continuous models in the last years $[10,21,42,44,54,60]$.

Since in such simulations the optical properties under operating conditions are of interest, they usually couple a drift-diffusion transport model with the calculation of the electronic states using a linear combination of atomic orbitals (LCAO) [28]. The method of choice seems to be emprirical tight binding due to its good compromise between accuracy and computational cost [37, 38, 46]. Since tight-binding is based on a localized basis associated with the atomic positions, it is very easy to include alloy fluctuations in the simulation. It has only to be considered that in the case of periodic structures like QWs a sufficiently big supercell has to be constructed to exclude spurious effects due to the periodically repeated random pattern. A compromise between supercell size and computational effort has to be chosen, therefore.

The multiscale setup and simulation flow employed in [14] is shown in Figure 3 Such an approach can be categorized as an overlap scheme, since the atomistic model is used as alternative way to describe the local density of states and optical properties on the same domain where the drift-diffusion model is applied. For computational reasons we only linked the models, instead of self-consistently coupling them. The figure shows a typical band edge profile, indicating also the relevant aspects (injection across potential barriers, non-radiative losses, radiative recombination) which have to be considered in order to gain useful insight into device operation. In this example, the interest has been in the effect of random alloy fluctuations on the radiative recombination of a single $\mathrm{QW}$. Therefore, a region around the $\mathrm{QW}$ of a single QW LED has been modelled by an atomistic structure with supercell size of $10 \times 10 \mathrm{~nm}^{2}$, and with a random distribution of indium atoms in the QW. Strain has been calculated using continuous elasticity [51] and valence force field (VFF) [20], and the desired operating point has been reached by selfconsistently solving the Schrödinger equation in $\mathrm{k} \cdot \mathrm{p}$ approximation [22] and the Poisson/drift-diffusion equations in 1D. The resulting electrostatic potential has been projected onto the atomic positions and the electronic states and from these the optical transitions have been obtained from the tight binding Hamiltonian. This allowed to estimate the radiative recombination parameter in presence of alloy fluctuations, and in particular its wavelength dependence. A very good agreement with experiment has been found for the latter [14]. These simulations have been performed with the tibercad software [16], which has been one of the first attempts to provide a generic multiscale framework for device simulation. 
(a)

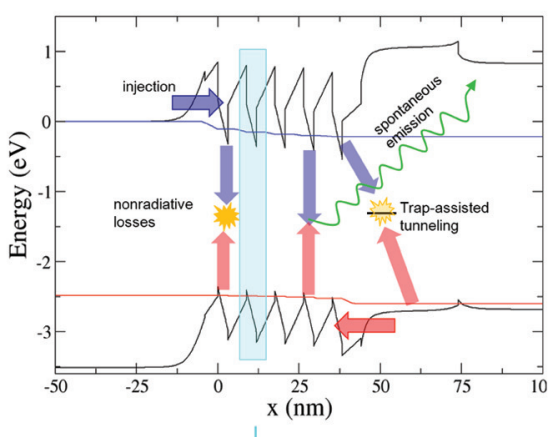

(b)

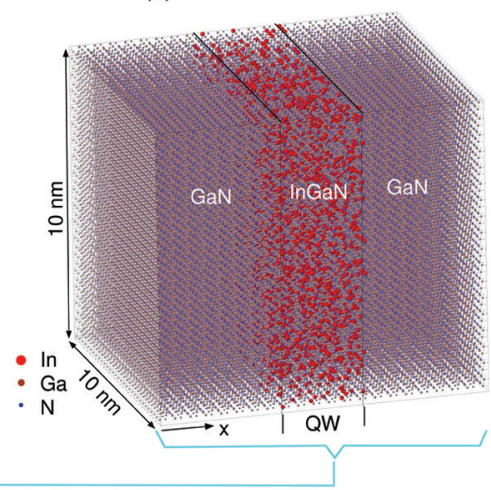

(c)

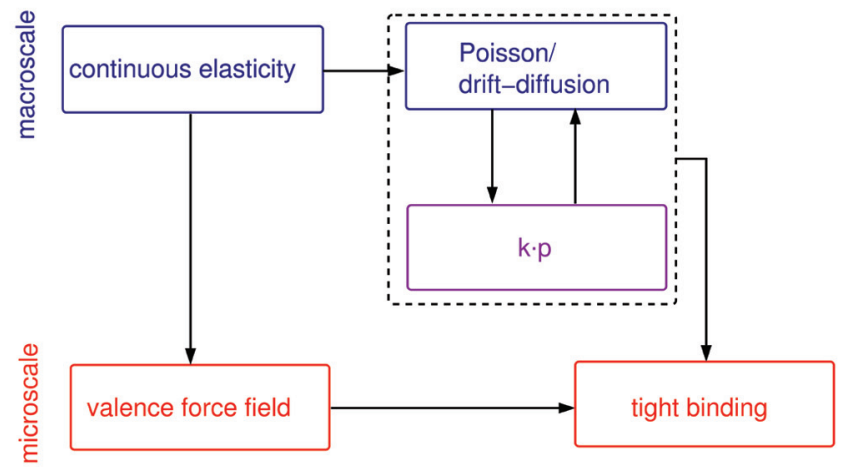

Figure 3 Scheme of the nitride LED simulation setup. Typical band edge profile, showing conduction and valence band ans the quasi Fermi levels resulting from a semi-classical driftdiffusion simulation, and indicating the important processes influencing device efficiency (a). The atomistic structure with random indium distribution and $10 \mathrm{~nm}$ in-plane supercell size (b). The models involved and the simulation flow (c). The tight binding model is solved only for the operating point of interest, and after having solved selfconsistently the Poisson/drift-diffusion equations and the continuous Schrödinger equation based on $\mathrm{k} \cdot \mathrm{p}$ approximation.

Note that the described simulation setup is computationally quite intensive, although it employs "only" an empirical tight binding model. Also, due to the statistical randomness of the single structures, a relatively big super-cell has to be chosen (containing roughly 100,000 atoms in this example), a sufficient number of states and k-points needs to be considered, and all calculations have to be done on a statistic ensemble of 30 or more random samples. Therefore, the numerical solvers like the eigenvalue solver have to be optimised [52], 
and parallelization schemes have to be employed. For example, different random samples and k-points can be calculated in parallel. This shows that that high performance computing (HPC) infrastructures can be of great importance for reasonable multiscale simulations.

\subsection{Coupling of Transport Models}

There are many situations where the active region of a device would be more adequately described by a microscopic transport model. The same example of II-nitride QW structures can be used here. As seen in Figure 3a, the active region in such devices has a complicated potential profile forming a number of barriers hindering carrier transport and sandwiching deep potential wells. The parameter of interest which has to be provided by a numerical simulation is the quantum efficiency $(\mathrm{QE})$, which depends on the different recombination processes, injection and capture efficiency and carrier leakage, i.e. on an interplay of transport and recombination processes, in a system that has a mixture of confined and delocalized (bulk-like) electronic states. While a semi-classical description like drift-diffusion can give a good representation of device characteristics in terms of fitting e.g. current-voltage or efficency curves $[19,50]$, a microscopic model relying on less empirical fitting parameters would certainly provide more insight into the details of the physical processes.

Since non-equilibrium Green's functions formalism (NEGF) is nowadays very popular and often used for transport calculations in nanostructures, there has been growing interest in applying it to optoelectronic devices, including LEDs $[3,5,6,13,56,59]$. While a ballistic NEGF calculation can be set up relatively easily, it is unfortunately very challenging to include all relevant scattering mechanisms consistently. Implementation of e.g. electron-phonon and electron-photon scattering is well established, but less work has been done on defect-mediated [4] and Auger scattering, which however are of paramount importance in nitride LEDs. In any case, a NEGF including scattering becomes computationally extremely intensive so that it is desirable to apply it to only the relevant portion of the device and couple it to a model like drift-diffusion describing the macroscopic part of the device where a local equilibrium and diffusive transport can be assumed.

Such an approach has been followed in [39] for a resonant tunneling diode and in [47] for a MOSFET, where NEGF and drift-diffusion has been solved self-consistently in a coupled setup. The scheme of this type of setup is presented in Figure 4. Note that such a setup can be a mixture of bridging 


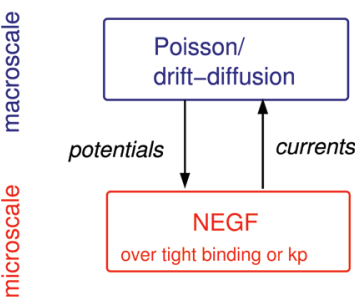

(a)

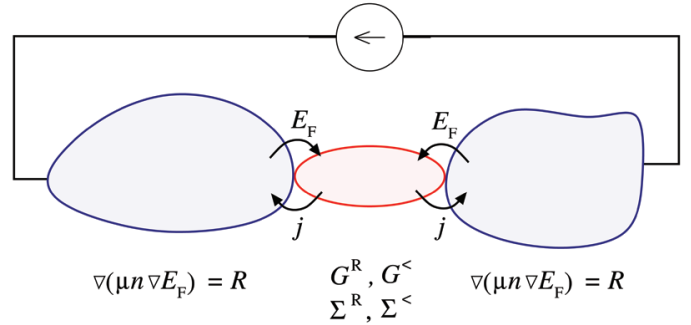

(b)

Figure 4 Scheme of a coupled NEGF/drift-diffusion simulation. The drift-diffusion model provides the quasi Fermi level $E_{\mathrm{F}}$ as boundary condition to NEGF, which in turn gives the current density $j$ at the interface back to the drift-diffusion model. In (b) some of the relevant quantities are indicated: the continuity equations for the drift-diffusion model with the carrier density $n$, mobility $\mu$ and recombination $R$ (the latter two modeling the effect of scattering), and the Green's functions $G^{\mathrm{R},<}$ and self-energies $\sum^{\mathrm{R},<}$ for NEGF.

and overlapping, in the sense that different quantities might be linked or coupled differently between different scales.

Here we show as a conceptual example a preliminary setup linking drift-diffusion and NEGF, which allows to estimate the resistivity of the GaN barriers between two QWs of a multi-QW LED. This is of interest because the conductivity in the barriers in a semi-classical drift-diffusion picture is proportional to the carrier densities, which decreases exponentially towards the top of the barriers. At usual injection currents this leads to quasidiscontinuities in the quasi Fermi levels which manifest in the current-voltage characteristics as an additional voltage drop. As a consequence, the simulated knee voltage is overestimated, which is quite typical for drift-diffusion based LED simulations. An example is given in Figure 5.

For the calculation of the ballistic current, we extract a barrier including a portion of the preceding and following QW. Using the electrostatic potential of the drift-diffusion simulation, we construct the Hamiltonian, or based on $\mathrm{k} \cdot \mathrm{p}$ or tight binding, and then calculate the ballistic electron current using NEGF, with quasi Fermi levels as boundary conditions for carrier injection. From the resulting current and the quasi Fermi levels at the two boundaries of the quantum mechanical domain, we extract the effective resistivity of the barrier, which we can compare with the semi-classical case. In this particular case we have assumed a small amount of 5\% of indium in the GaN barrier in order to gain some insight into the effect of random alloy fluctuations on the transmission, similar to the example before. Since the semi-classical 

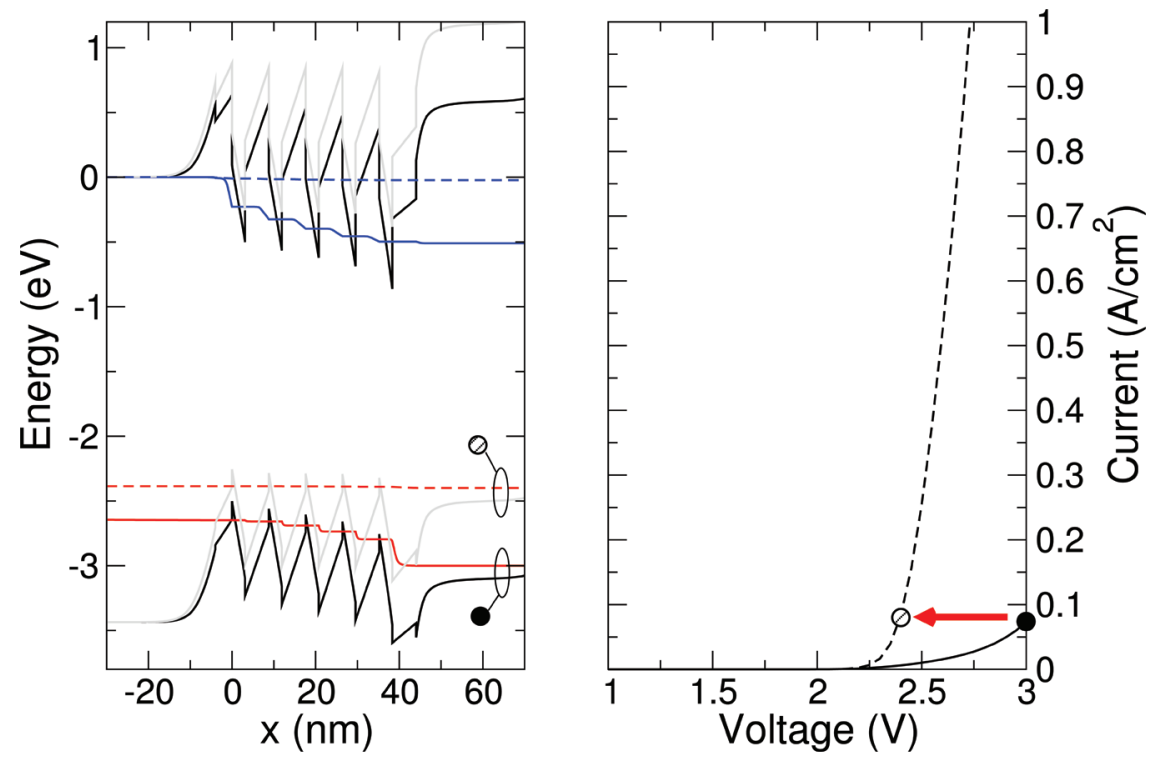

Figure 5 Current-voltage characteristics for a InGaN/GaN MQW LED resulting from a drift-diffusion simulation with two different conductivities in the barrier regions (right panel, solid line: conductivity proportional to carrier density, dashed line: additional artifical conductivity of $10^{-5} \mathrm{~S} / \mathrm{cm}$ ). The left panel shows the band edge profiles and quasi Fermi levels for both cases at $80 \mathrm{~mA} / \mathrm{cm}^{2}$.

drift-diffusion and ballistic NEGF models are linked by means of boundary conditions as shown in Figure 4, we can categorize this example as a bridging scheme with respect to electronic transport.

The domain for the NEGF calculation and the resulting transmissions for different Hamiltonians $(\mathrm{k} \cdot \mathrm{p}$ compared to different random structures for tight binding) are shown in Figure 6. The resistivities extracted from the results are: $15 \Omega \cdot \mathrm{cm}^{2}$ for standard drift-diffusion, $1.33 \Omega \cdot \mathrm{cm}^{2}$ for $\mathrm{k} \cdot \mathrm{p}, 0.28-1.26 \Omega \cdot \mathrm{cm}^{2}$ for different random configurations and tight binding, which is comparable to the numbers used for the results in Figure 5.

Note that this example hides a number of difficulties. In particular, the treatment of the contacts in NEGF requires special attention in order to prevent spurious effects [40]. This has not been considered for the above example, so that the results can at most give some rough idea, which is enough for our purpose of illustrating the basic ideas. A more detailed implementation of the scheme, including self-consistent coupling, has been presented recently in [32]. 


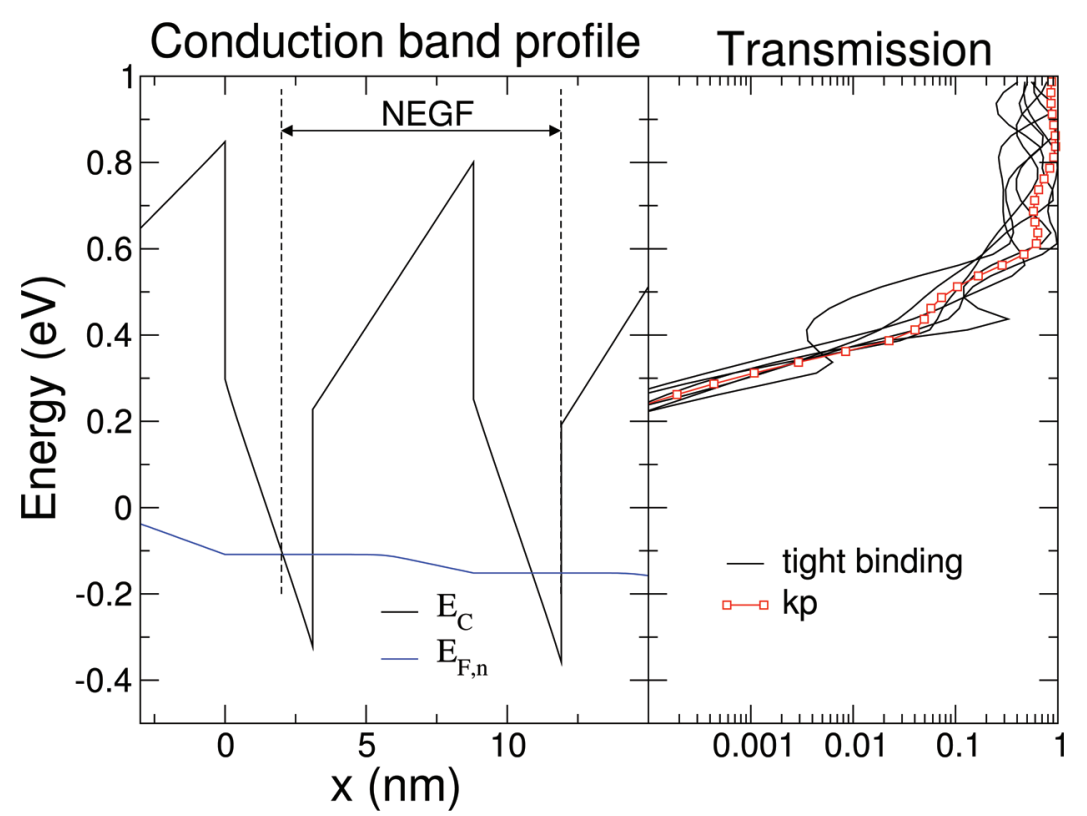

Figure 6 Detail of conduction band profile and electron quasi Fermi level around one barrier (left panel). The domain where NEGF has been applied is highlighted. The right panel shows the energy resolved transmisson, inidcating a strong influence of the random nature of the distribution of a small amount of indium atoms in the barrier.

\subsection{Prospects of Multiscale Simulations for Photovoltaics}

Multiscale simulation setps can be expected to play an increasing role in photovoltaics, not only for research purposes but also for engineering and optimisation of application ready technologies. In particular next generation cell concepts are structurally and functionally of a complexity that necessarily requires multiscale approaches. For example, organic solar cells are usually based on an absorber which is a blend of acceptor and donor molecules forming a complex morphology on the $10 \mathrm{~nm}$ scale. The interfaces between the constituent organic materials are of paramount importance and need detailed description. Here, multiscale approaches can be employed to couple microscopic models for the processes happening at or across the interfaces - like exciton dissociation, charge transfer, energetic alignments or recombinations to macroscopic transport models. This route is followed for example in [23, 27]. A different approach has been followed in [65], where a Monte Carlo simulation on real morphology has been used to obtain macroscale 
transport parameters, while in [49] Monte Carlo has been coupled with a field-theoretic microscopic model. A third approach has been adopted in [31] by embedding a realistic morphology in a subdomain of an effective media based drift-diffusion model in order to study specific effects related to carrier traps at the material interfaces.

Beside organic solar cells, also other concepts will benefit of multiscale simulation approaches. Quantum dot based devices for example, which are of interest for intermediate band solar cells [43], lend themselves to a modeling strategy where the optical and electronic properties of the quantum dot arrays are obtained by microscopic models. This has been done for example in [55], where a Monte Carlo model has been combined with a density matrix based microscopic description of the quantum dot absorber layer.

Interest in multiscale approaches is also growing for the modeling of the optical properties in solar cells, in particular when plasmonic structures are involved. Some recent works include [7], which is applying a multiscale solver for the electromagnetic problem, [45] where a quantum mechanical model for absorption is coupled to a classical electromagnetic solver, and in [41] where a ray tracing is linked with finite difference time domain (FDTD) calculations.

For particular concepts like hot electron solar cells, in addition a multiscale treatment of phonon related properties might become of interest. To the authors knowledge there has been no activity so far in this field related to photovoltaics.

\section{Conclusions}

Multiscale approaches for the simulation of optical, electronic and thermal properties in electronic devices are gaining more and more importance not only for basic research, but also for applied device engineering. A detailed description of modern devices requires microscopic models to accurately simulate all relevant processes in the active device regions, which however normally are too computationally expensive to be used on the whole device comprising macroscopic access regions. Therefore, and in view of device optimisation and application-oriented use of such simulation models a multiscale coupling with macroscopic models is required. This will combine the best of each model on every scale, and take profit of the long standing experience in industry with macroscale models like e.g. drift-diffusion.

In this article we underpinned the importance of multiscale approaches and showed that interest has been growing world wide during the years, 
evidenced by the public funding of specific research programs. Some simulation examples have been shown, where a multiscale model can provide valuable additional insight into device behaviour. Next generation solar cell devices can be expected to benefit particularly from multiscale modeling, and different approaches have already been presented in literature or started to be implemented.

The future will show to what extent multiscale simulations will be able to enter optoelectronic device industry as a helpful tool for device development and optimization. This will depend not least on successful integration of multiscale approaches in industry grade simulation environments, from both usability and computational points of view. Definition of common understandings, terminology and standards will help in this process so that different research fields can mutually benefit fom their respective research and model implementions.

\section{Acknowledgements}

The author gratefully acknowledges Aldo Di Carlo, Alessandro Pecchia, Alessio Gagliardi, Desiree Gentilini and Fabio Sacconi for inspiring discussions and the COST association for supporting European research activity in multiscale solar cell modeling and characterisation in COST Action MP1406 "MultiscaleSolar".

\section{References}

[1] https://www.nsf.gov

[2] http://www.goldbeck-consulting.com/home.html/27120/25020.html

[3] U. Aeberhard. Theory and simulation of quantum photovoltaic devices based on the non-equilibrium green's function formalism. Journal of Computational Electronics, 10(4):394-413, 2011.

[4] U. Aeberhard. Quantum-kinetic theory of defect-mediated recombination in nanostructure-based photovoltaic devices. In Materials Research Society Symposium Proceedings, volume 1493, pages 91-96, 2013.

[5] U. Aeberhard. Quantum-kinetic theory of steady-state photocurrent generation in thin films: Coherent versus incoherent coupling. Physical Review B - Condensed Matter and Materials Physics, 89(11):115303, 2014. 
[6] U. Aeberhard. Simulation of nanostructure-based and ultra-thin film solar cell devices beyond the classical picture. Journal of Photonics for Energy 4(1):042099, 2014.

[7] H. Ameri, M. Fallahpour, M. Li, and W. C. Chew. An efficient numerical method to analyze nanoplasmonic structures. In Radio Science Meeting (Joint with AP-S Symposium), 2015 USNC-URSI, pages 64-64, July 2015.

[8] G. Anciaux, O. Coulaud, and J. Roman. High performance multiscale simulation for crack propagation. In Parallel Processing Workshops, 2006. ICPP 2006 Workshops. 2006 International Conference on, pages 8 pp. $-480,2006$.

[9] A. Arnold and A. Jüngel. Multi-scale modeling of quantum semiconductor devices. In A. Mielke, editor, Analysis, Modeling and Simulation of Multiscale Problems. Springer-Verlag, Berlin, 2006.

[10] M. Auf der Maur, D. Barettin, A. Pecchia, F. Sacconi, and A. Di Carlo. Effect of alloy fluctuations in InGaN/GaN quantum wells on optical emission strength. In Numerical Simulation of Optoelectronic Devices (NUSOD), 2014 14th International Conference on, pages 11-12. IEEE, 2014.

[11] M. Auf der Maur, G. Penazzi, G. Romano, F. Sacconi, A. Pecchia, and A. Di Carlo. The multiscale paradigm in electronic device simulation. IEEE Transactions on Electron Devices, 58(5):1425-1432, May 2011.

[12] M. Auf der Maur, M. Povolotskyi, F. Sacconi, A. Pecchia, and A. Di Carlo. Multi-scale simulation of MOS systems based on high- $\kappa$ oxides. Journal of Computational Electronics, 7(3):398-402, 2008.

[13] M. Auf der Maur. Multiscale approaches for the simulation of ingan/gan leds. Journal of Computational Electronics, 14(2):398-408, 2015.

[14] M. Auf der Maur, Alessandro Pecchia, Gabriele Penazzi, Walter Rodrigues, and Aldo Di Carlo. Efficiency drop in green InGaN/GaN light emitting diodes: The role of random alloy fluctuations. Phys. Rev. Lett., 116:027401, Jan 2016.

[15] M. Auf der Maur, Alessandro Pecchia, Gabriele Penazzi, Fabio Sacconi, and Aldo Di Carlo. Coupling atomistic and continuous media models for electronic device simulation. Journal of Computational Electronics, 12(4):553-562, 2013.

[16] M. Auf der Maur, Michael Povolotskyi, Fabio Sacconi, Alessandro Pecchia, Giuseppe Romano, Gabriele Penazzi, and Aldo Di Carlo. Tibercad: towards multiscale simulation of optoelectronic devices. Optical 
and Quantum Electronics, 40:1077-1083, 2008. 10.1007/s11082-0099272-7.

[17] Ivo Babuska. Homogenization and its application. mathematical and computational problems. Proc. of the $3^{\text {rd }}$ Symposium on Numerical Solution of Partial Differential Equations, pages 89-116, 1976.

[18] Elizabeth Brunk and Ursula Rothlisberger. Mixed quantum mechani$\mathrm{cal} /$ molecular mechanical molecular dynamics simulations of biological systems in ground and electronically excited states. Chemical Reviews, 115(12):6217-6263, 2015. PMID: 25880693.

[19] M. Calciati, M. Goano, F. Bertazzi, M. Vallone, X. Zhou, G. Ghione, M. Meneghini, G. Meneghesso, E. Zanoni, E. Bellotti, G. Verzellesi, D. Zhu, and C. Humphreys. Correlating electroluminescence characterization and physics-based models of InGaN/GaN LEDs: Pitfalls and open issues. AIP Advances, 4(6):067118, 2014.

[20] D. Camacho and Y. M. Niquet. Application of keating's valence force field model to non-ideal wurtzite materials. Physica E, 42:1361-1364, 2010.

[21] Miguel A. Caro, Stefan Schulz, and Eoin P. O'Reilly. Theory of local electric polarization and its relation to internal strain: Impact on polarization potential and electronic properties of group-III nitrides. Phys. Rev. B, 88:214103, Dec 2013.

[22] S. L. Chuang and C. S. Chang. k - p method for strained wurtzite semiconductors. Physical Review B, 54:2491-2504, 1996.

[23] T. Costa, D. Foster, and M. Peszynska. Domain decomposition for heterojunction problems in semiconductors. Lecture Notes in Computer Science (including subseries Lecture Notes in Artificial Intelligence and Lecture Notes in Bioinformatics), 8969:92-101, 2015. cited By 0.

[24] S. Datta. Electronic Transport in Mesoscopic Systems. Cambridge University Press, 1995.

[25] A. F. de Baas and R. Lula, editors. What makes a material function? Let me compute the ways. Publications Office of the European Union, 2015.

[26] Carlo de Falco, Emilio Gatti, Andrea L. Lacaita, and Riccardo Sacco. Quantum-corrected drift-diffusion models for transport in semiconductor devices. Journal of Computational Physics, 204(2):533-561, 2005.

[27] Carlo de Falco, Matteo Porro, Riccardo Sacco, and Maurizio Verri. Multiscale modeling and simulation of organic solar cells. Computer Methods in Applied Mechanics and Engineering, 245246:102-116, 2012. 
[28] A. Di Carlo. Microscopic theory of nanostructured semiconductor devices: beyond the envelope-function approximation. Semiconductor Science and Technology, 18:1, 2003.

[29] Y. Efendiev, T. Hou, and V. Ginting. Multiscale Finite Element Methods for Nonlinear Problems and their Applications. Comm. Math. Sci. 2:553-589, 2004.

[30] J. A. Elliott. Novel approaches to multiscale modelling in materials science. International Materials Reviews 56(4):207-225, 2011.

[31] A. Gagliardi, M. Auf Der Maur, D. Gentilini, F. Di Fonzo, A. Abrusci, H. J. Snaith, G. Divitini, C. Ducati, and A. Di Carlo. The real tio2/htm interface of solid-state dye solar cells: Role of trapped states from a multiscale modelling perspective. Nanoscale, 7(3):1136-1144, 2015. cited By 5.

[32] J. Geng, P. Sarangapani, E. Nelson, C. Wordelman, B. Browne, Kubis T., and G. Klimeck. Multi-Scale, Multi-Physics NEGF Quantum Transport for Nitride LEDs. In Proceedings of th $16^{\text {th }}$ International Conference on Numerical Simulation of Optoelectronic Devices, pages 107-108, 2016.

[33] J. Guo, S. Datta, M. Lundstrom, and M. P. Anantram. Towards MultiScale Modeling of Carbon Nanotube Transistors. eprint arXiv:condmat/0312551, December 2003.

[34] Z. Hassan, N. Allec, Li Shang, R.P. Dick, V. Venkatraman, and Ronggui Yang. Multi-scale thermal analysis for nanometer-scale integrated circuits. Computer-Aided Design of Integrated Circuits and Systems, IEEE Transactions on, 28(6):860-873, June 2009.

[35] M. F. Horstemeyer. Multiscale modeling: A review. In J. Leszczyski and M. K Shukla, editors, Practical Aspects of Computational Chemistry: Methods, Concepts and Applications, page 87135. Springer, 2009.

[36] T. J. R. Hughes. Multiscale Phenomena: Green's functions, the Dirichletto-Neumann formulation, subgrid scale models, bubbles and the origins of stabilized methods. Comput. Methods Appl. Mech. Engrg., 127: 387-401, 1995.

[37] J. M. Jancu, F. Bassani, F. Della Sala, and R. Scholz. Transferable tight-binding parametrization for the group-III nitrides. Applied Physics Letters 81(25):4838, 2002.

[38] J.-M. Jancu, R. Scholz, F. Beltram, and F. Bassani. Empirical spds ${ }^{\star}$ tight-binding calculation for cubic semiconductors; General method and material parameters. Physical Review B 57(11), 1998. 
[39] Gerhard Klimeck. Quantum and semi-classical transport in nemo 1-d. Journal of Computational Electronics 2(2-4):177-182, 2003.

[40] T. Kubis and P. Vogl. Assessment of approximations in nonequilibrium green's function theory. Physical Review B - Condensed Matter and Materials Physics, 83(19), 2011. cited By 11.

[41] Claude Leiner, Susanne Schweitzer, Volker Schmidt, Maria Belegratis, Franz-Peter Wenzl, Paul Hartmann, Ulrich Hohenester, and Christian Sommer. Multi-scale simulation of an optical device using a novel approach for combining ray-tracing and fdtd. Proc. SPIE 8781, Integrated Optics: Physics and Simulations, pages 87810Z-87810Z-9, 2013.

[42] M. Lopez, A. Pecchia, M. Auf der Maur, F. Sacconi, G. Penazzi, and A. Di Carlo. Atomistic simulations of $\mathrm{InGaN} / \mathrm{GaN}$ random alloy quantum well LEDs. Phys. Status Solidi C, 2014.

[43] A. Luque and A. Mart. Increasing the efficiency of ideal solar cells by photon induced transitions at intermediate levels. Physical Review Letters 78(26):5014-5017, 1997. cited By 1268.

[44] M. Lopez, F. Sacconi, M. Auf der Maur, A. Pecchia, and A. Di Carlo. Atomistic simulation of InGaN/GaN quantum disk LEDs. Optical and Quantum Electronics 44(3):89-94, 2012.

[45] L. Meng, C. Yam, Y. Zhang, R. Wang, and G. Chen. Multiscale modeling of plasmon-enhanced power conversion efficiency in nanostructured solar cells. Journal of Physical Chemistry Letters 6(21):4410-4416 2015. cited By 1.

[46] Fabiano Oyafuso, Gerhard Klimeck, R. Chris Bowen, and Timothy B. Boykin. Atomistic electronic structure calculations of unstrained alloyed systems consisting of a million atoms. Journal of Computational Electronics 1(3):317-321, 2002.

[47] A. Pecchia, M. Auf der Maur, and A. Di Carlo. Coupling lengthscale from drift-diffusion to non equilibrium green's functions. In $15^{\text {th }}$ International Workshop on Computational Electronics (IWCE), pages 45-46, 2012.

[48] J. Peng, Q. Chen, N. Wong, L. Y. Meng, C. Y. Yam, and G. H. Chen. New Multi-Scale Simulation Framework for Next-Generation Electronic Design Automation with Application to the Junctionless Transistor. e-print arXiv:cond-mat/1207.3765, July 2012.

[49] Anton Pershin, Sergii Donets, and Stephan A. Baeurle. A new multiscale modeling method for simulating the loss processes in polymer solar cell nanodevices. The Journal of Chemical Physics, 136(19), 2012. 
[50] J. Piprek. III-Nitride LED efficiency droop models: A critical status review. In 13th International Conference on Numerical Simulation of Optoelectronic Devices, NUSOD 2013, pages 107-108, 2013.

[51] M. Povolotskyi and A. Di Carlo. Elasticity theory of pseudomorphic heterostructures grown on substrates of arbitrary thickness. Journal of Applied Physics, 100:063514, 2006.

[52] W. Rodrigues, A. Pecchia, M. Auf der Maur, and A. Di Carlo. A comprehensive study of popular eigenvalue methods employed for quantum calculation of energy eigenstates in nanostructures using gpus. Journal of Computational Electronics 14(2):593-603, 2015.

[53] R. E. Rudd and J. Q. Broughton. Concurrent coupling of length scales in solid state systems. physica status solidi (b) 217(1):251-291, 2000.

[54] Stefan Schulz, Miguel A. Caro, Conor Coughlan, and Eoin P. O'Reilly. Atomistic analysis of the impact of alloy and well-width fluctuations on the electronic and optical properties of InGaN/GaN quantum wells. Phys. Rev. B, 91:035439, Jan 2015.

[55] A.V. Semichaevsky and H.T. Johnson. Carrier transport in a quantum dot solar cell using semiclassical and quantum mechanical models. Solar Energy Materials and Solar Cells, 108:189-199, 2013. cited By 5.

[56] Akshay Shedbalkar, Zhelio Andreev, and Bernd Witzigmann. Simulation of an indium gallium nitride quantum well light-emitting diode with the non-equilibrium green's function method. physica status solidi (b), 253(1):158-163, 2016.

[57] L. Shifren, C. Ringhofer, and D. K. Ferry. A wigner function-based quantum ensemble monte carlo study of a resonant tunneling diode. IEEE Transactions on Electron Devices 50(3):769-773, 2003.

[58] S. Sinha and Kenneth E. Goodson. Review: Multiscale thermal modeling in nanoelec-tronics. International Journal for Multiscale Computational Engineering 3(1):107-133, 2005.

[59] S. Steiger, R. G. Veprek, and B. Witzigmann. Electroluminescence from a quantum-well led using negf. In Proceedings - 2009 13th International Workshop on Computational Electronics, IWCE 2009, page 5091112, 2009.

[60] Daniel P. Tanner, Miguel A. Caro, Eoin P. O'Reilly, and Stefan Schulz. Atomistic analysis of the electronic structure of m-plane ingan/gan quantum wells: Carrier localization effects in ground and excited states due to random alloy fluctuations. physica status solidi (b), pages n/a-n/a, 2015. 
[61] Elaine Taylor, Paul R. Edwards, and Robert W. Martin. Colorimetry and efficiency of white leds: Spectral width dependence. physica status solidi (a) 209(3):461-464, 2012.

[62] Jeffrey Y. Tsao, Mary H. Crawford, Michael E. Coltrin, Arthur J. Fischer, Daniel D. Koleske, Ganapathi S. Subramania, G. T. Wang, Jonathan J. Wierer, and Robert F. Karlicek. Toward Smart and Ultra-efficient Solid-State Lighting. Advanced Optical Materials, 2(9):809-836, 2014.

[63] Giovanni Verzellesi, Davide Saguatti, Matteo Meneghini, Francesco Bertazzi, Michele Goano, Gaudenzio Meneghesso, and Enrico Zanoni. Efficiency droop in ingan/gan blue light-emitting diodes: Physical mechanisms and remedies. Journal of Applied Physics, 114(7):-, 2013.

[64] C. Weber, A. Wacker, and A. Knorr. Density-matrix theory of the optical dynamics and transport in quantum cascade structures: The role of coherence. Phys. Rev. B, 79:165322, Apr 2009.

[65] F. Wei, L. Liu, L. Liu, and G. Li. Multiscale modeling and simulation for optimizing polymer bulk heterojunction solar cells. IEEE Journal of Photovoltaics 3(1):300-309, 2013. cited By 6.

[66] E. Weinan, B. Engquist, X. Li, W. Ren, and E. Vanden-Eijnden. Heterogeneous Multiscale Methods: A Review. Communications in Computational Physics, 2:367-450, 2007.

[67] S. P. Xiao and T. Belytschko. A bridging domain method for coupling continua with molecular dynamics. Comput. Methods Appl. Mech. Eng. 193(17-20):1645-69, 2004/05/07.

[68] Y. Xu and N. R. Aluru. Multiscale electrostatic analysis of silicon nanoelectromechanical systems (NEMS) via heterogeneous quantum models. Phys. Rev. B, 77:075313, 2008.

[69] S. Yamakawa, S. Goodnick, S. Aboud, and M. Saraniti. Quantum corrected full-band cellular Monte Carlo simulation of AlGaN/GaN HEMTs. Journal of Computational Electronics, 3(3-4):299-303, 2004.

[70] S. Zhang, R. Khare, Q. Lu, and T. Belytschko. A bridging domain and strain computation method for coupled atomistic-continuum modelling of solids. International Journal for Numerical Methods in Engineering, 70:913-933, 2007. 


\section{Biography}

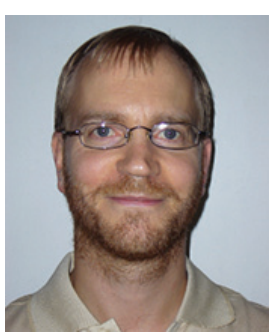

M. Auf der Maur, Ph.D. in 2008 with University of Rome "Tor Vergata" in Electronic Engineering. His research interests are the development of multi-scale/multi-physics simulation models and software for electronic and optoelectronic devices, and the simulation of devices based on such models. He has long experience in simulation of III-nitride devices, organic solar cells and LEDs and dye solar cells. Since 2008 he is researcher with the Dept. of Electronics engineering of University of Rome "Tor Vergata". 
